\title{
Proceeding
}

9th INSHS International Christmas Sport Scientific Conference, 4-6 December 2014. International Network of Sport and Health

Science. Szombathely, Hungary

\section{Relationship between isokinetic muscle strength and 100 meters finswimming time}

\author{
VLADIMIR KUNITSON $\triangle$, KRISTJAN PORT, KIRSTI PEDAK \\ Institute of Health Sciences and Sport, Tallinn University, Tallinn, Estonia
}

\begin{abstract}
Kunitson, V., Port, K., \& Pedak, K. (2015). Relationship between isokinetic muscle strength and 100 meters finswimming time. J. Hum. Sport Exerc., 9(Proc1), pp.S482-S489. Finswimming is a sport where athlete uses one big monofin to produce propulsion. The purpose of this study was to describe relationship between isokinetic strength of different muscle groups and 100 meters finswimming time. Study questions was: what muscle groups have relationship with finswimming time. Methods: 17 international level monfin swimmers, 9 male (age 16,9 $\pm 1,8$ yrs., height $180,0 \pm 10.0 \mathrm{~cm}$., weight $76.0 \pm 6.9 \mathrm{~kg}$ ) and 8 female finswimmers ( age 17,6 $\pm 4,7$ yrs., height 170,0 $\pm 10.0 \mathrm{~cm}$., weight $68,6 \pm 13,5 \mathrm{~kg}$ ) performed 100 meters monofin surface swim and where also tested within a two days on isokinetic dynamometer (knee, hip and trunk extensors and flexors at angular speeds of $60 \% \mathrm{sec}$ ). Force data at angles from 0-90 degrees with 10 degree step were used for correlation. Results: There was a strong correlation $(p<0.05)$ between swimming time and knee extensors $\left(r=-0.82\right.$ at angle $\left.50^{\circ}\right)$, hip flexors $\left(r=-0.70\right.$ at angle $\left.10^{\circ}\right)$ and trunk flexors $(r=-0.77$ at angle $20^{\circ}$ ) in male swimmers. Surprisingly, we did not find corresponding correlations among female finswimmers. Conclusion: Current study found relationship between strength of main muscle groups and swimming speed only in male swimmers. It is expected that strength plays a role in swimming time, but the lack of relationship in female swimmers suggests at the need to introduce a measure of swimming effectiveness instead of pure resultant time. The proposed hypothesis is further supported by the high level of competence among study participants (incl. multiple European champion, and several finalists). Key words: MONOFIN SWIMMING, KNEE EXTENSORS, HIP FLEXORS, TRUNK FLEXORS.
\end{abstract}

\footnotetext{
Corresponding author. Tallinn University, Institute of Health Sciences and Sport, Räägu 49, Tallinn, Estonia E-mail: vladimir.kunitson@gmail.com 9th INSHS International Christmas Sport Scientific Conference, 4-6 December 2014. International Network of Sport and Health Science. Szombathely, Hungary. JOURNAL OF HUMAN SPORT \& EXERCISE ISSN 1988-5202

(c) Faculty of Education. University of Alicante doi:10.14198/jhse.2015.10.Proc1.42
} 


\section{INTRODUCTION}

Finswimming is a popular cyclic sport which is performed either on the surface or underwater. Compared to the classical swimming finswimmers make use of the fins that alter the load profile of the muscular work. In the classical swimming major propulsion forces are generated by the muscles of the upper body limbs. However, in Finswimming athletes avoid using upper extremities and try to keep them in a streamline position throughout the distance to minimize the resistance from the drag and they utilize mainly lower limbs alongside with trunk muscles to produce propulsion movement channelling the forces via the surface of the monofin (Rejman et al., 2003). Legs in a coordinated effort with trunk and hip muscles produce a kicking movement which is similar to the classical dolphin style swimming leg kicking technique. Two types of fins and corresponding sports disciplines are in use: bi-fin with two independent fins for each leg, and a single monofin that combines work of both legs in one common movement. Current study is about monofin swimming.

Comparing monofin swimming techniques between genders both at novice and expert levels it has been found that both women and novice in general bend their knees more than men and expert fin swimmers (Gautier et al., 2004). Cited study did not provide explanations, and we propose that women and novice swimmers lack in the strength compared to men and expert fin swimmers. Linkage with the strength of the legs and swimming speed in monofin swimming has been demonstrated by holding the amplitude of the movement stable and avoiding errors of the distal part of the fin (Rejman, 2013).

To our knowledge there is only one study in finswimming where the relationship between muscular strength and swimming outcomes have been tested (Kunitson et al., 2013). Study found a strong correlation ( $\mathrm{r}=-$ $0.88, p<0.05$ ) between isometric knee extensor strength and finswimming time. Relationship between knee flexors and finswimming time was not established. It is reasonable to infer that the hip and trunk flexors and extensors are working in conjunction with the knee extensors during the monofin swimming. According to Bali finswimming movement starts from the hip (Bali et al., 2001).

Studies of functional status, recruitment profiles and operational patterns of muscles in finswimming are rare and the field deserves more attention. Muscular strength needs to be developed in a sport specific manner and supported with specific strength exercises (Maglischo, 1993). Therefore, to plan an effective strength and conditioning training one needs to know what muscles are involved and how these are recruited in finswimming.

Isokinetic muscle testing is used in the swimming and has for example been shown to be reliable for establishing association between the functional abilities of quadriceps muscle group and the swimming speed (Dalamitros et al., 2014). Also, a correlation has been found between the isokinetic strength of knee extensors and the flutter kicking in short distance swimming of 25 and 50 yards (Mookerjee et al., 1995). Nevertheless, the relationship of functional status of various muscles with the swimming performance has yet to be studied extensively.

Isokinetic strength measurements provides an opportunity to study the relationship between a defined muscle group and finswimming time. In current study we used isokinetic measurements to establish whether there is a relationship between the monofin swimming speed and the functional status of extensor and flexor muscles running over the knee and the hip joints, as well as the trunk muscles. 


\section{MATERIAL AND METHODS}

\section{Participants}

17 elite level finswimmers were asked to participate voluntarily. 8 of them were female (age 17,6 $\pm 4,7 \mathrm{yrs}$., height $170 \pm 10 \mathrm{~cm}$., body weight 68,6 $\pm 13,5 \mathrm{~kg}$.) and 9 male (age 16,9 $\pm 1,8$ yrs., height $180 \pm 10 \mathrm{~cm}$., body weight $76 \pm 6,9 \mathrm{~kg}$.). 1 of participants was European champion, 1 World record holder, 2 were European championship finalists and one World Cup finalists, and the remaining athletes were at least medal winners in the national championships.

\section{Procedures}

Two day experimental protocol consisted of two stages. Day one: $100 \mathrm{~m}$ swimming in a short course pool, filmed with the underwater camera moving along the distance and split times of every 25 meters were recorded; Day two: strength measurements using isokinetic dynamometer (Humac Norm, Computer Sports Medicine, Inc. Stroughton MA, USA). The knee, hip (both sides) and trunk extensors and flexors were tested in accordance with the recommended protocol in the dynamometer's user manual. Using the "gravity correction" procedure limbs were weighted to negate gravity effect of the weight of the limbs. Concentric test at velocities $60^{\circ} / \mathrm{sec}$ was used. Subjects performed 4 warm-up trials and after 20 seconds of recovery followed the 5 repetitions maximal test trials. 12-15 minutes rest period were used between testing of different muscle groups. Force data at angles from 0-90 degrees with 10 degree step were used for analysis.

\section{Analysis}

Due to the independent tests for left and right legs data was summed prior to further analysis to present a joint effort in monofin swimming. Data analysis consisted of descriptive and parametric inferential statistics (Pearson correlation) at $p<0.05$. As the Pearson's correlation coefficient is sensitive to the data distribution and specifically for outliers, data was inspected for consistency before analysis.

\section{RESULTS}

Average swimming time in seconds, for the groups of male and female athletes correspondingly, are given in the Table 1.

Table 1. Mean + SD values of 100 meters finswimming times (seconds)

\begin{tabular}{cc}
\hline Gender & Mean time \pm SD \\
\hline Male & $44.40 \pm 1.94$ \\
Female & $48.00 \pm 3.10$ \\
\hline
\end{tabular}

In the case of the female swimmers data from the isokinetic tests failed to demonstrate any meaningful correlation with the swimming outcome. Therefore the following analysis concentrates only on the data from the male swimmers. Also, search of the correlation between the swimming speed and isokinetic force generation profiles of various muscle groups revealed a notable relationship with trunk and hip flexors and knee extensors only.

Figure 1 describes the relationship of force generation in knee extensors over the movement trajectory from 0 to 90 degrees at the speed of $60 \% \mathrm{sec}$. We found that force produced in knee extensors muscle group 
had a significant correlation with the swimming speed only at a range of motion at 40, 50 and 60 degrees (355,33 $\pm 117,02 \mathrm{Nm} ; 407 \pm 108,76 \mathrm{Nm} ; 426,33 \pm 60,87 \mathrm{Nm}$ accordingly) (see also Table 2 for correlation size estimates).

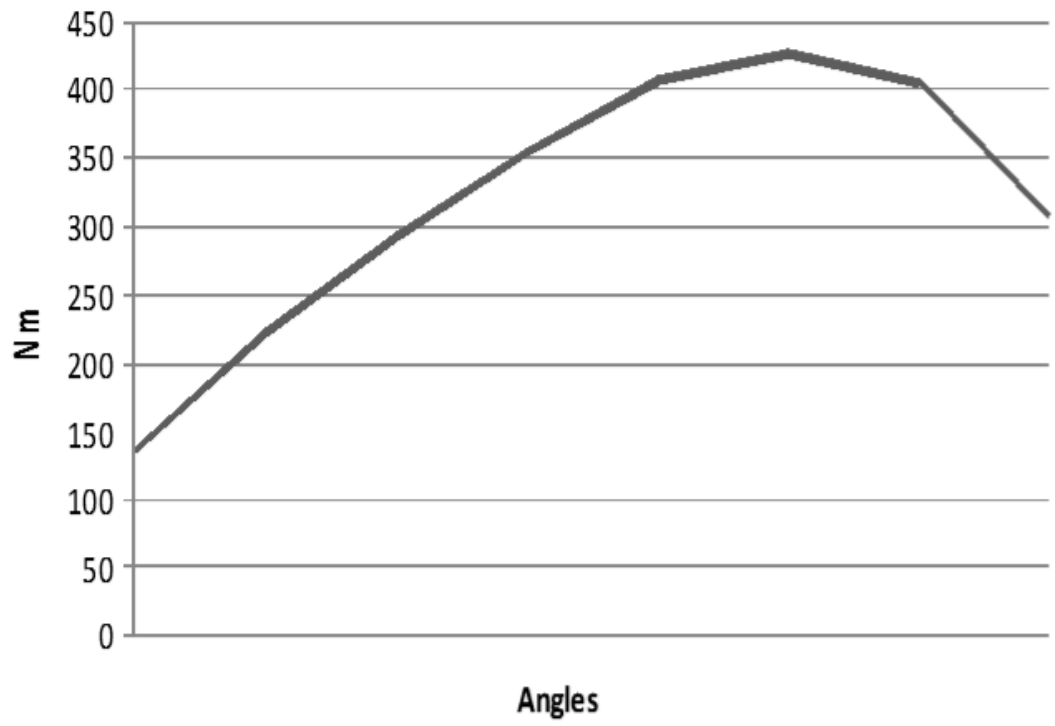

Figure 1. Average knee extensor force generation profile at $60 \% \mathrm{sec}$ over 0 to 90 degrees for male swimmers

Likewise, for trunk flexors (see Figure 2) significant correlation was established at $10^{\circ}(276,78 \pm 86,58 \mathrm{Nm})$ to $20^{\circ}(316,78 \pm 58,62 \mathrm{Nm})$ degrees, and again at $70^{\circ}(161,11 \pm 40,19 \mathrm{Nm})$ (see also Table 2 for correlation size estimates).

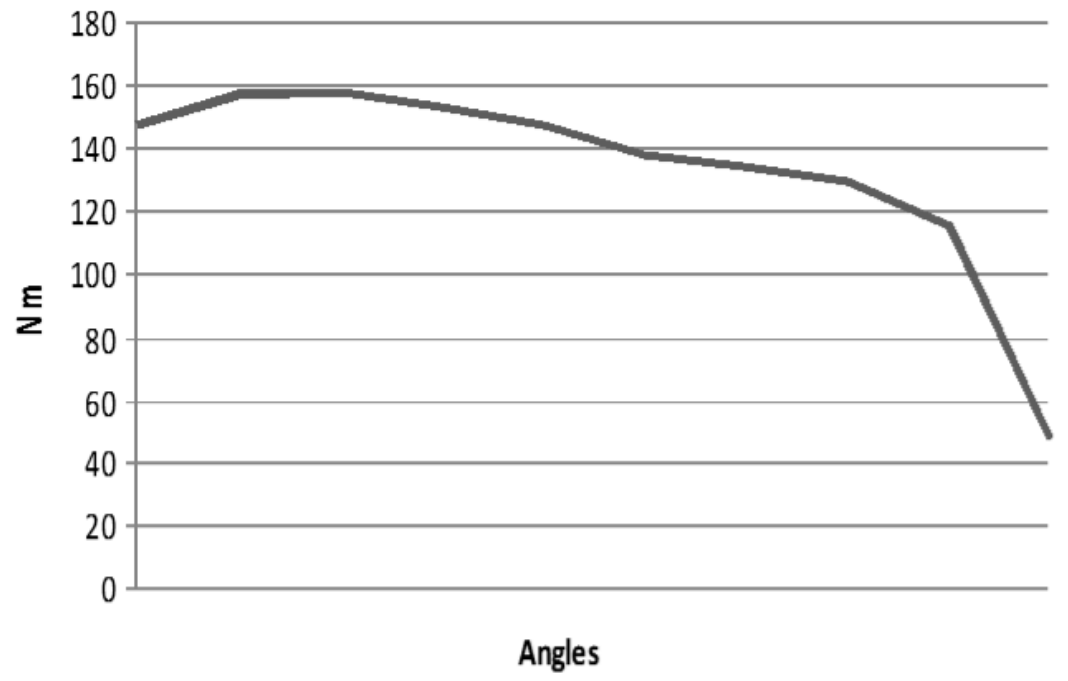

Figure 2. Average trunk flexors force generation profile at $60 \% \mathrm{sec}$ over 0 to 90 degrees for male swimmers. Angle at -10 describes the tendency to overextend before a forceful upper body forward and down movement 
Thirdly, for hip flexors (Figure 3) the swimming speed variation correlated with the variation of force generation ability in male swimmers at $0^{\circ}$ to $40^{\circ}(156,56 \pm 31,54 \mathrm{Nm} ; 158,11 \pm 30,34 \mathrm{Nm} ; 152,78 \pm 30,25$ $\mathrm{Nm} ; 147,44 \pm 28,72 \mathrm{Nm} ; 137,67 \pm 29,55 \mathrm{Nm}$ correspondingly) (see also Table 2 for correlation size estimates).

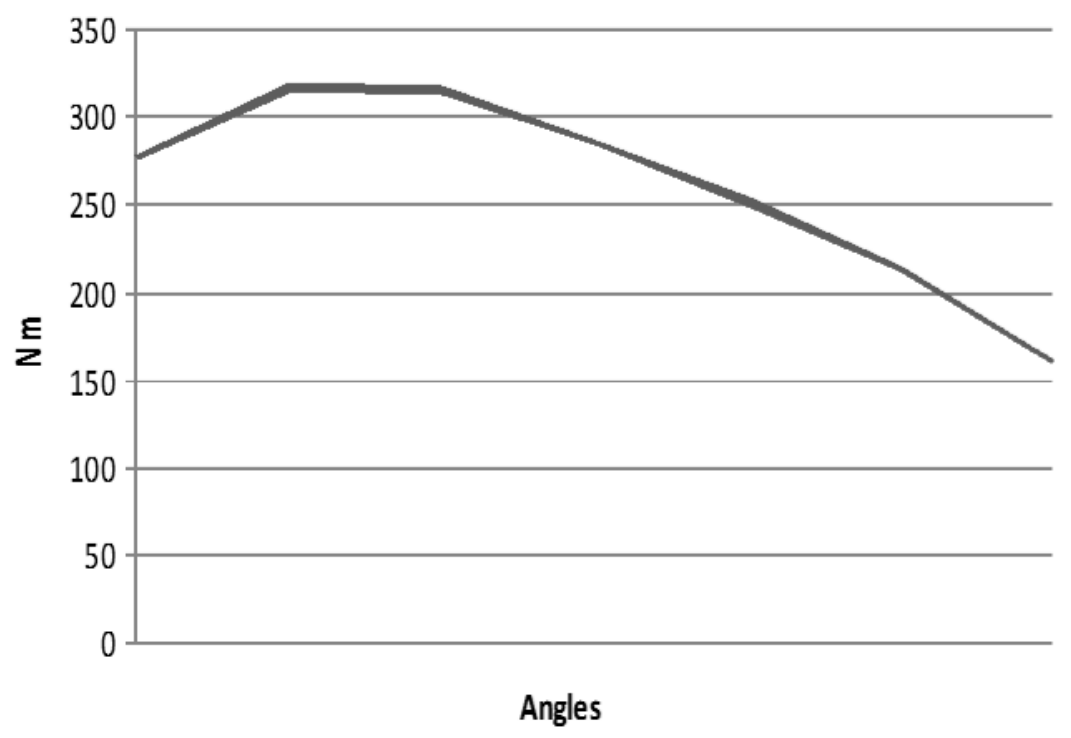

Figure 3. Average hip flexor force generation profile at $60 \% \mathrm{sec}$ over 0 to 90 degrees for male swimmers

Corresponding sizes of correlation between the swimming speed and the capacity of isometric force generation at various degrees of range of movements in the three depicted muscle groups display certain distance dependency as is shown in Table 2.

Table 2. Correlation between isokinetic muscle strength and finswimming times.

\begin{tabular}{cllll}
\hline & $25 \mathrm{~m}$ & $50 \mathrm{~m}$ & $75 \mathrm{~m}$ & $100 \mathrm{~m}$ \\
\hline Knee extensors & & & & \\
\hline $40^{\circ}$ & $-794^{*}$ & $-719^{*}$ & $-709^{*}$ & $-782^{*}$ \\
$50^{\circ}$ & $-824^{* *}$ & $-768^{*}$ & $-742^{*}$ & $-811^{* *}$ \\
$60^{\circ}$ & $-733^{*}$ & & & \\
\hline Hip Flexors & & & & \\
\hline $10^{\circ}$ & $-702^{*}$ & $-685^{*}$ & $-693^{*}$ & \\
$20^{\circ}$ & $-706^{*}$ & & & \\
$70^{\circ}$ & $-737^{*}$ & & & \\
\hline Trunk Flexors & & & $-688^{*}$ \\
\hline $0^{\circ}$ & $-676^{*}$ & & $-699^{*}$ \\
$10^{\circ}$ & $-728^{*}$ & & \\
$20^{\circ}$ & $-770^{*}$ & & \\
$30^{\circ}$ & $-756^{*}$ & & \\
$40^{\circ}$ & $-741^{*}$ & &
\end{tabular}




\section{DISCUSSION}

The aim was to investigate relationship between the isokinetic muscle strength and finswimming time. We found that in the male swimmers there is a significant relationship between the ability to generate force at certain angles of isokinetic tests and swimming speed. Specifically, we found the relationship to exist only for the particular muscle groups, namely for the trunk and hip flexors and knee extensors. We did not find a similar pattern of the muscle recruitment effect on the swimming speed among the female swimmers which is in accordance with our earlier study findings (Kunitson et al., 2013). Also, it has been previously found that isokinetic muscle strength and finswimming time were found only in male swimmers in the study of Miyashita and Kanehisa (1979), as well as in (Dalamaitros et al., 2014) who found knee extension peak torque to be negatively correlated ( $r=-0.58)$ in boys, but not in girls. Oshita et al. 2013 investigated gender difference in aerobic contribution to surface performance in finswimming. They found using critical swimming speed that aerobic performance capacity contributed to the surface performance in the events from 200 to 1500 meters in female athletes but only at distances 800 and 1500 in male athletes. It is expected that strength plays a role in swimming time, but the lack of relationship in female swimmers suggests at the need to introduce a measure of swimming effectiveness instead of pure resultant time.

However this does not indicate that the swimming speed among the female swimmers is not dependent on the ability to generate force, and we hypothesize that this phenomena may be also related to relatively lower resistance due to lower swimming speed that results in less pronounced strength or vice versa. Whatever the case may be, we need further studies to understand the phenomena.

In the case of male swimmers we found that the force generation of had a relatively high explanatory part in swimming speed for knee extensors $(r 2=-0.68)$, hip flexors $(r 2=-0.50)$ and trunk flexors $(r 2=-0.59)$. The finding is partly supported by an earlier study that found the knee extensors to have a correlation $(r=-0.88)$ with monofin swimming result at 200m surface swimming among male finswimmers (Kunitson et al., 2013).

Surprisingly we did not find relationship between the knee flexors, hip and trunk extensors and the swimming speed. Again, this does not imply to the lack of the relevance of these muscle groups in swimming outcome, but instead encourages to develop the sensitivity and specificity of study protocols. It is reasonable to expect that the hip extensors are related to the efficiency of finswimming upbeat segment. As during the upbeat movement straight leg must be brought up and no knee flexing is allowed. That is the possible reason why the knee flexors did not show any correlation with resultant speed. Moving the hip up during the down beat movement must therefore result because of the knee straightening out and not as the trunk unbending. We understand that this is the reason why the force of the trunk extensor muscles did not have any correlation with swimming speed.

Correlation of muscle strength at particular different joint angles only among different muscle groups is either explained with the limited movement range of the joints in knee, hip and trunk as can be seen in Figures 4 and 5 depicting swimmers in water at various stages of movement. Another explanation may be dominance of anthropometric optimum of force generation at the specific angles of movement that fundamentally constrains the swimming technique. In either cases further assessment of the relative importance of the dynamic properties of the force generation in the various joints and muscle assemblies is justified. One must not forget that current depiction of correlation does not imply per se to causation, but will help to pinpoint out the elements that are relevant in the building of the model for the monofin swimming performance analysis and for prerequisites of training. 


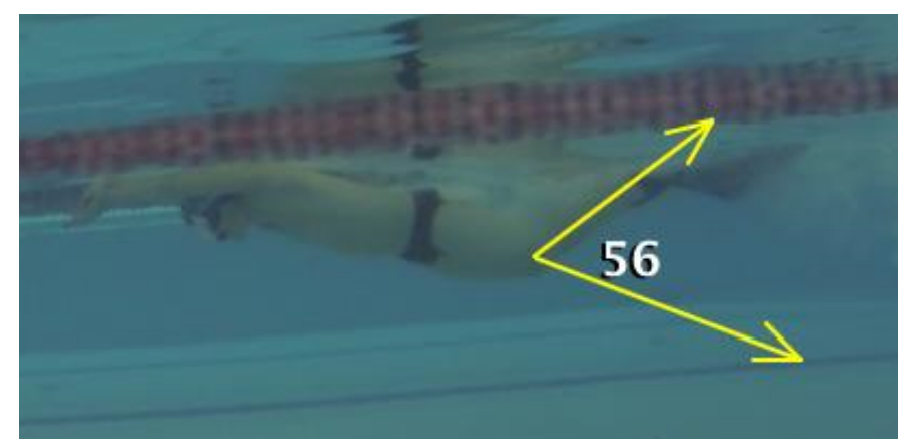

Figure 4. Movement range of the joints in knee

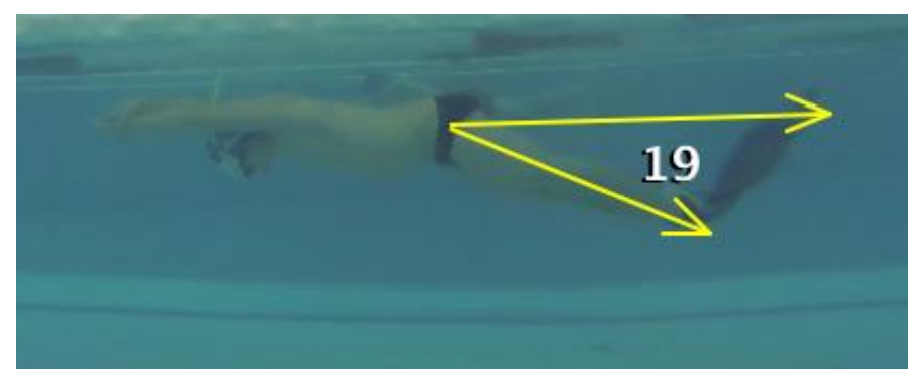

Figure 5. Movement range of the joints in knee

In current study we found that the movement range of the lower limbs and the trunk that are used in the monofin swimming coincide with the angles at which the force correlated with the swimming speed. This may give ground for the selection of movement range for the strength training exercises. The importance of using the stroke specific exercises have been highlighted also by Maglischo (1993).

\section{CONCLUSIONS}

Current study found relationship between swimming speed and the force of one extensor and two flexor muscles groups only among men and only at certain angles of movement range.

\section{REFERENCES}

1. Baly, L., Favier, D., \& Durey, A. (2001). Finswimming tehnical description by 3D kinematic study. Arch Physiol Biochem., 1(109), pp.67.

2. Dalamitros, A.A., Manou, V., \& Pelarigo, J.G. (2014). Labaratory-based tests for swimmers: methodology, reliability, considerations and relationship with front-crawl performance. J. Hum. Sport Exerc., 9(1), pp.172-187.

3. Gautier, J., Baly, L., Zanone, P.G., \& Watier, B. (2004). A kinematic study of finswimming at surface. J Sports Sci Med., 3, pp.91-95.

4. Kunitson, V., Rannama, I., \& Port, K. (2013). Relationship between isokinetic muscle strenght and finswimmin time. LASE, 4(1), pp.55-61.

5. Maglischo, E.W. (2003). Swimming Fastest. Human Kinetics, 3.

6. Miyashita, M., Kanehisa, H. (1979). Dynamic peak torque related to age, sex and performance. Res Quart., 50(2), 249-255. 
7. Mookerjee, S., Bibi, K.W., Kenney, G.A., \& Cohen, L. (1995). Relationship between isokinetic strength, flexibility and flutter kicking speed in female collegiate swimmers. J. Strength Cond. Res., 9(2), pp.71-74.

8. Oshito, K., Ross, M., Koizumi, K., Tsuno, T., \& Yano, S. (2013). Gender difference of aerobic contribution to surface performance in finswimming: analysis using critical velocity method. Asian $\mathrm{J}$ Sport Med., 4, pp.256-262.

9. Rejman, M. (2013). Analysis of relationships between the level of errors in leg and monofin movement and stroke parameters in monofin swimming. J Sports Sci Med., 12, pp.171-181.

10. Rejman, M., Colman, V., \& Persyn, U. (2003). The method of assessing the kinematics and dynamics of single fin movements. Hum Movement Sci., 2(8), pp.54-62. 